\title{
Concomitant Existence of Bilateral Adrenal Adenomas. To Operate or Not?
}

Christos DAMASKOS ${ }^{a, b *}$, Nikolaos GARMPIS ${ }^{b, c *}$, Dimitrios DIMITROULIS Anna GARMPI ${ }^{d}$, Paraskevi FARMAKI ${ }^{\text {, }}$ Alexandros PATSOURAS ${ }^{\dagger}$, Vasilili Epameinondas GEORGAKOPOULOUg, Georgios KYRIAKOS ${ }^{\mathrm{h}}$, Lourdes Victoria QUILES-SANCHEZi, Athanasios SYLLAIOSi, Aliki LIAKEAk, Evangelos DIAMANTIS'

aRenal Transplantation Unit, Laiko General Hospital, Athens, Greece

${ }^{b}$ N.S. Christeas Laboratory of Experimental Surgery and Surgical Research, Medical School, National and Kapodistrian University of Athens, Athens, Greece

'Second Department of Propedeutic Surgery, Laiko General Hospital, Medical School, National and Kapodistrian University of Athens, Athens, Greece

dFirst Department of Propedeutic Internal Medicine, Laiko General Hospital, Medical School, National and Kapodistrian University of Athens, Athens, Greece

eFirst Department of Pediatrics, Agia Sofia Children's Hospital, Medical School,

National and Kapodistrian University of Athens, Athens, Greece

fSecond Department of Pulmonology, Sotiria General Hospital, Athens, Greece

sDepartment of Pulmonology, Laiko General Hospital, Athens, Greece

hSeccion de Endocrinologia y Nutrition, Hospital General Universitario Santa Lucia,

Cartagena, Spain

'Centro de Salud Jesús Marín, Murcia, Spain

jFirst Department of Surgery, Laiko General Hospital, Medical School, National and Kapodistrian University of Athens, Athens, Greece

kFirst Department of Pathology, Medical School,

National and Kapodistrian University of Athens, Athens, Greece

'Academic Department of Internal Medicine, Endocrinology Unit,

General Oncology Hospital of Kifisia Agioi Anargyroi,

National and Kapodistrian University of Athens, Athens, Greece

*Authors with equal contribution

\section{Address for correspondence:}

Damaskos Christos, MD, MSc, PhD

Renal Transplantation Unit, Laiko General Hospital, N.S. Christeas Laboratory of Experimental Surgery and Surgical Research, Medical School, National and Kapodistrian University of Athens, Athens, Greece

Postal address: 17 Agiou Thoma Street, 11527, Athens, Greece

Tel: +30 6948467790; email: $x \_d a m a s k o s @ y a h o o . g r$ 
-ABSTRACT

Nowadays, through the wide use of both magnetic resonance imaging and computed tomography, the diagnosis of adrenal incidentaloma is becoming increasingly frequent. Pheochromocytomas are neuroendocrine tumors which produce catecholamine, and they are characterized by headaches, palpitations, sweating and hypertension. Aldosterone-secreting adrenal cortical adenomas can cause various metabolic and cardiovascular diseases due to aldosterone excess. Our aim is to present a rare case of a concomitant existence of pheochromocytoma in the right adrenal and a functioning adrenal cortical adenoma in the left, worthwhile mentioning since the appearance of these two entities in different location in the same patient is unprecedented. The treatment remains challenging.

Keywords: adrenal incidentaloma, pheochromocytoma, non-functioning adenoma, primary aldosteronism, aldosteronoma.

\section{INTRODUCTION}

1 random radiological finding of an adrenal lesion greater than $1 \mathrm{~cm}$ in diameter, conducted for other medical purpose, defines adrenal incidentaloma (AI) (1). It is of paramount importance to check patients with Al for pheochromocytoma, hyperaldosteronism (if hypertension exists), hypercortisolism and malignancy (2). The most common etiology of an $\mathrm{Al}$ is the non-functioning adenoma, and its prevalence is estimated at $80 \%$ (3). The incidence of pheochromocytoma itself is estimated to approximately 1:100.000 (4). The prevalence of primary aldosteronism in hypertensive patients is about $4 \%$ and it constitutes as the most common etiology of secondary hypertension (5). Bilateral adrenal hyperplasia is the main cause of primary aldosteronism, followed by other causes such as aldosteronoma (6). Concerning resistant hypertension, the prevalence of primary aldosteronism increases to $14-21 \%$ (7). The definition of resistant hypertension includes the elevation of systolic and diastolic blood pressure above the levels of $140 \mathrm{~mm} \mathrm{Hg}$ and $90 \mathrm{~mm} \mathrm{Hg}$, respectively, which is not improved using three different antihypertensive drugs of different classes (8).

Herein, we describe the rare case of two distinct adrenal masses in location, defined as a left aldosteronoma and a histopathologically proven right pheochromocytoma.

\section{CASE PRESENTATION}

A 57-year-old Caucasian female patient with hypertension, headaches, flushing, palm sweating and palpitations, which lasted a few hours and regressed with orally administrated nifedipine (calcium channel blocker) $10 \mathrm{mg}$, referred to the emergency department. Three months before, an abdominal ultrasound (US) was conducted for annual precautionary purposes. US showed a compact, round, right adrenal mass. Subsequently, a computed tomography (CT) and Magnetic resonance imaging (MRI) of lower abdomen took place, which confirmed the existence of a well bordered lesion measuring $9 \times 6.7 \times 2.5 \mathrm{~cm}$ in the right adrenal. There was neither extension of the lesion to adjacent organs nor the surrounding fatty tissue was depicted as abnormal. The mass presented internal cystic degeneration and intense enhancement of compacts after intravenous administration of contrast media. The CT also demonstrated a lesion above the left kidney, with dimensions of $3.11 \times 1.7 \times 1 \mathrm{~cm}$. The lesion was homogenous, round-shaped not consistent to malignancy. An Al was revealed. A ${ }^{123}$-metaiodobenzylguanidine (MIBG) scintigraphy revealed a large, circumscribed uptake region in the anatomical position of the right adrenal gland. These findings were consistent with pheochromocytoma, and the patient was admitted to hospital for further examination. Her whole medical history contained diabetes type 2, osteoporosis and there were no hormonal symptoms. The vitals were normal except for blood pressure 150/90 mm Hg and the clinical findings on physical examination did not reveal any abnormality. The 24-hour urine metanephrine levels were elevated (4.700 mcg/day - normal <350 mcg/24 hours). Aldosterone/renin ratio was normal, and the $1 \mathrm{mg}$ overnight dexamethasone suppression test were also normal. The patient was treated with phenoxybenzamine as well as atenolol in order to block $\alpha-1$ vascular receptors and to prepare for surgical treatment. The patient underwent right laparoscopic adrenalectomy with clear surgical margins (R0) (Figure 1A). The surgical specimen measured $10.5 \times 7.1 \times 2.7 \mathrm{~cm}$ and weighed $103 \mathrm{~g}$ (Figure 1B). The histological examination demonstrated an 
encapsulated yellowish and brownish-hemorrhagic tumor of a soft composition. The cells varied in shape and size and were arranged in nests. They were polygonal, with eosinophilic cytoplasm and hyperchromasia was also noticed. Fibrous tissue was observed between the nests. The immunohistochemical staining was positive for Synaptophysin (+), Chromogranin (+), NSE $(+)$ and negative for S-100 (-), Melan-A (-), HMB-45 (-), Inhibin (-). The cell proliferation index was below $1 \%$ in tumor cells. The pathologist confirmed the clinical diagnosis, and this lesion was characterized as pheochromocytoma (Figure 2).

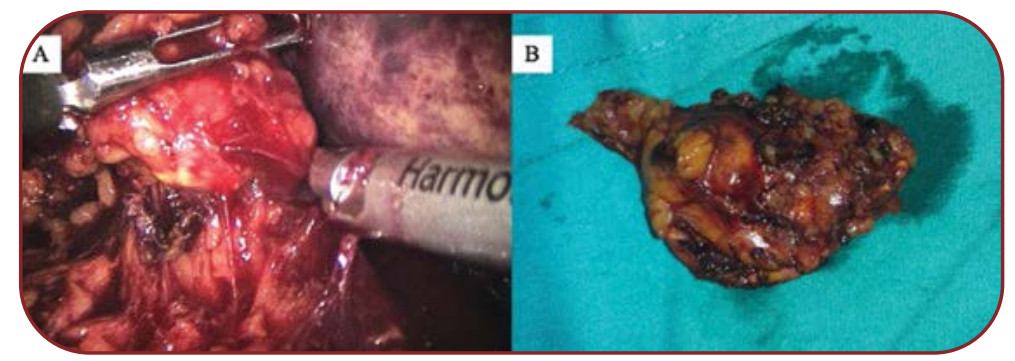

FIGURE 1. Surgical operation. A: Laparoscopic right adrenalectomy; B: Surgical specimen

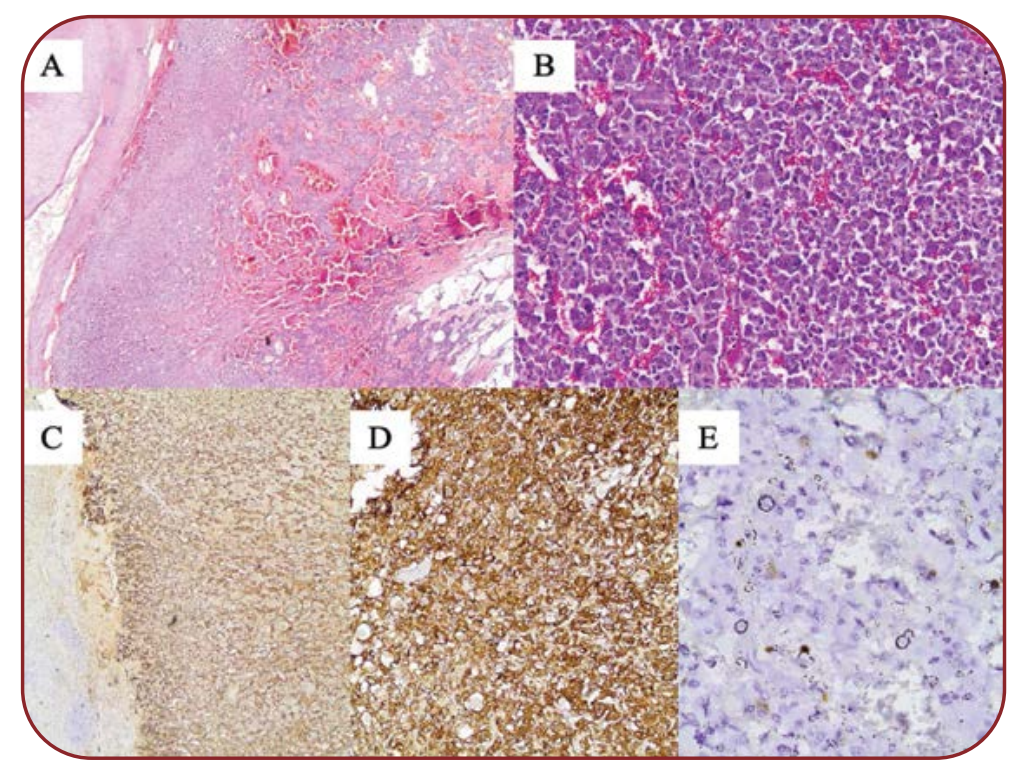

FIGURE 2. Histopathological findings. A: Pheochomocytoma consisting of small nests (zellballen) and solid sheets of polygonal shaped cells in a rich vascular network, with capsular and adipose tissue invasion and a compressed rim of adrenal cortex on the upper right (hematoxylin-eosin, original magnification x20); B: Pheochromocytoma composed of cells of various nuclear pleomorphism, with finely granular basophilic cytoplasm and round oval nuclei with prominent nucleolus (hematoxylin-eosin, original magnification x200); C, D: Strong positive cytoplasmic staining of pheochromocytoma cells for chromogranin and synaptophysin, respectively (immunohistochemistry, original magnification x100); E: Ki67 proliferation index shows a low proliferative activity (approximately 1\%) (immunohistochemistry, original magnification $\mathrm{x} 400$ )
Four days postoperatively symptoms disappeared and the levels of urinary catecholamines returned to normal. Blood pressure levels were normal $(138 / 85 \mathrm{~mm} / \mathrm{Hg})$ and a follow-up program in the endocrinology department was recommended to the patient. The patient showed clinical improvement in blood pressure levels and all other above-mentioned symptoms disappeared. Also, the laboratory examinations of catecholamine levels remained normal. Imaging follow-up did not reveal any changes in left adrenal lesion measurements (Figures 3, 4 A, B). Unfortunately, the patient was lost to follow-up after two years.

Eleven years later, the patient reappeared due to the presence of edemas, weight gain, muscular cramps, and fatigue. The history of the left adrenal mass was already known. As a result, further evaluation was done. Regarding the vitals, only blood pressure was elevated at about 153/81 $\mathrm{mm} \mathrm{Hg}$. On physical examination, the patient had central obesity (BMI 30.8) with neither moon face nor abdominal cutaneous striae. Her personal history revealed type 2 diabetes mellitus (T2DM) and osteoporosis started more than 10 years ago. Firstly, urine and blood samples were obtained in order to check the functional status of the mass. Serum cortisol and adrenocorticotropic hormone (ACTH) were normal. Potassium levels were low $(2.7 \mathrm{mMol} / \mathrm{L})$, even though she did not receive any medication causing hypokalemia. After normalization of potassium levels, aldosterone concentration in plasma was $50 \mathrm{ng} / \mathrm{dL}$ and plasma rennin activity less than $0.5 \mathrm{ng} / \mathrm{mL} / \mathrm{h}$. Aldosterone/renin ratio was above limits and arterial gases demonstrated metabolic alkalosis. All findings were compatible to primary aldosteronism. Plasma- (43 pg/mL - normal 20-65 pg/mL) and 24-hour urinary $(270 \mathrm{mcg} /$ day - normal $<350 \mathrm{mcg} / 24$ hours) metanephrines fluctuated within the normal range. Similarly, normetanephrine levels were $60 \mathrm{pg} / \mathrm{mL}$ (normal $35-120 \mathrm{pg} / \mathrm{mL}$ ) in plasma and $310 \mathrm{mcg} /$ day (nor$\mathrm{mal}<450 \mathrm{mcg} / 24$ hours) in urine. Additionally, there were no abnormalities detected in serum creatinine, urea, electrolytes, and thyroid profile. Examinations revealed levels of urine free cortisol within normal limits and normal diurnal variation of serum cortisol levels. The levels of ACTH were between the normal ranges. Suppression of cortisol was observed after the $1 \mathrm{mg}$ overnight dexamethasone suppression test. Triple antihypertensive therapy was administrated to the patient, 


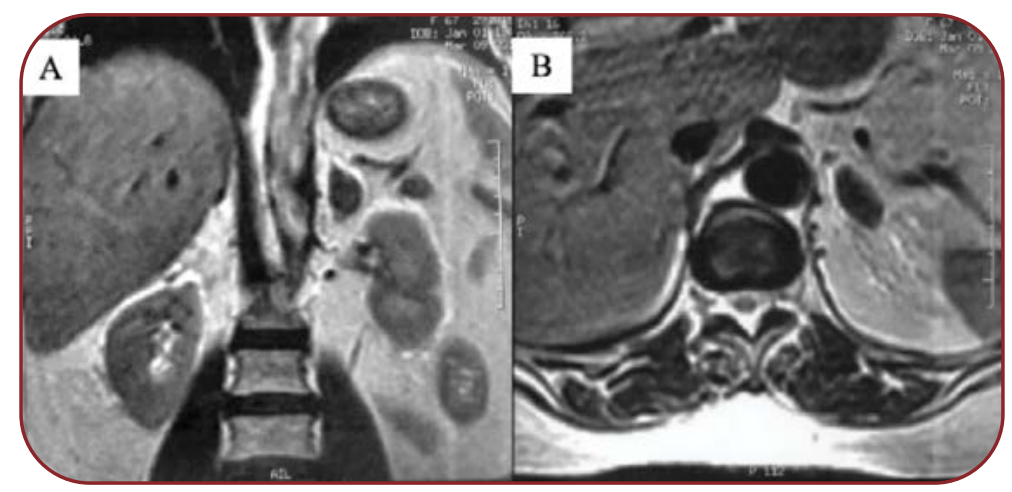

FIGURE 3. Postoperative imaging two years after right laparoscopic adrenalectomy. Follow-up magnetic resonance imaging indicated absence of right adrenal lesion measurements and no changes in left adrenal ones (T1 weighting)

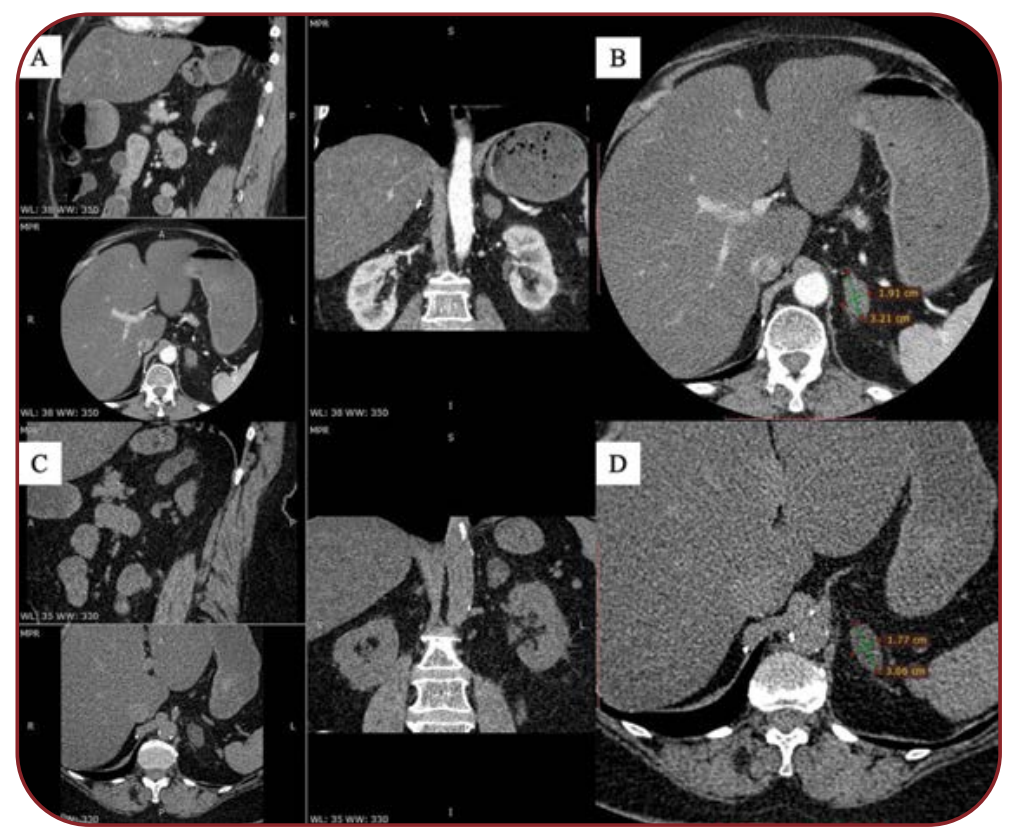

FIG URE 4. Postoperative imaging after right laparoscopic adrenalectomy. Follow-up computed tomography indicated absence of right adrenal lesion measurements and no changes in left adrenal ones. A: two years postoperatively; B: 14 years postoperatively

including spironolactone, nifedipine and captopril. No surgery was performed and a follow-up every three months was decided to monitor any change in the lesion and clinical response to antihypertensive treatment. After one year, the patient was healthy, her blood pressure was controlled within limits, no symptoms have been referred and no enlargement of the remaining adrenal has been observed (Figure 4 C, D).

\section{DISCUSSION}

ome authors have described cases of bilateral adrenal adenomas coexistence, or adenomas found in the same gland (9-16). Our case is worthwhile mentioning since the second lesion was clinically initially non-secreting. We should highlight that the use of new imaging techniques, including CT or US, render the adrenal masses a more frequent finding (17). The use of CT-guided or US-guided biopsy would both discover more of these cases and improve patients' clinical outcome since bilateral adrenalectomy and adrenal insufficiency would be prevented $(18,19)$.

The primary diagnostic objectives of an $\mathrm{Al}$ are either the definition of a malignancy or the existence of hormone secreting adrenal tumor (20). Imaging features in favor of benign adrenal lesion are less than 10 Hounsfield Units (HU) attenuation, fast washout of contrast medium in 10 to 15 minutes (20). Furthermore, round shape, size less than $4 \mathrm{~cm}$, unilateral location and homogeneous density are all factors consistent with benign adenomas $(21,22)$. As far as our patient is concerned, the possibility of malignancy seems remote since it is homogenous, small in size and round shaped. In addition, the absence of tumor development for one year minimizes the possibility of malignancy.

Pheochromocytoma usually appears to individuals between 40- to 60-year-old and it has an equal incidence in both sexes (24). It is associated to von Hippel Lindau (VHL) syndrome, multiple endocrine neoplasia type 2 (MEN2) and neurofibromatosis type 1 (NF1) or it appears sporadically (25). Familial pheochromocytomas occur in younger ages and adrenals are not the only affected organ (26). Genetic tests are required for the diagnosis (27). In our case, we cannot exclude the presence of familial pheochromocytoma. However, the older age of the patient and the location of the lesion only in the adrenals do not constitute the typical clinical presentation of this medical entity. Furthermore, the cost of genetic tests was another problem for the patient. The classic clinical presentation of pheochromocytoma includes anxiety, diaphoresis, paroxysmal headaches, and palpitations (28). The measurement of blood and urine catecholamines and their metabolites are used for the preoperative diagnosis (29). Computed tomography remains the primary imaging tool for pheochromocytoma, as in our case. It can be demonstrated as heterogeneous, homogeneous, necrotic, cystic, or even solid with attenuation on contrast CT more than $10 \mathrm{HU}$ (30). Magnetic resonance imaging (MRI) is used in patients with metastatic disease (29). We used the 123I-MIBG due to 
the big size of the primary lesion and its high risk for malignancy and possible metastasis; MIBG can be also useful for determining the exact location of the lesion, especially in the bigger ones $(31,32)$. In the microscope, cells could be polyhedral with dark granular cells with rounded nucleus (17). Preoperatively, it is of paramount importance to control blood pressure firstly with an a-adrenergic antagonist and subsequently use $\beta$-blockers. Last but not least important, removal of the entire gland after anti-hypertensive preoperative management is the primary treatment approach against pheochromocytoma. Follow-up requires yearly surveillance at least by lifetime measuring catecholamines and metanephrines in both plasma and urine for lifetime. Unfortunately, our patient was lost to follow-up until the re-appearance of symptoms. The use of CT or MRI is necessary during the follow-up period when laboratory tests reveal an abnormality (21).

The functional status of the tumor should be tested and evaluated as negative in order to diagnose a cortical adrenal lesion as non-functioning (33). The functional status is determined by the ratio of plasma aldosterone to plasma renin, urinary and plasma metanephrines, ACTH levels and dexamethasone suppression test. In individuals with $\mathrm{Al}$ in observation, a CT should be conducted in between three to six months and afterwards annually for two continuous years (3). Changes in the size of the lesion more than $0.8 \mathrm{~cm}$ or in the hormonal assessment during follow-up render surgery as an option (34). Non-functioning and unilateral tumors less than $4 \mathrm{~cm}$ with benign characteristics do not require surgical removal (35).

Our case was initially a non-secreting cortical adenoma, which turned into aldosteronoma. Hypokalemia with poor control of hypertension should raise suspicion for the diagnosis. However, it should be noted that normal levels of potassium are a frequent laboratory finding in this disease (36). A ratio of plasma aldosterone concentration to plasma rennin activity more than 30 is suggestive to primary aldosteronism (37). The main differential diagnosis is between bilateral adrenal hyperplasia and unilateral adenoma (6). The best way to diagnose it is the use of adrenal venous sampling. However, it is an invasive technique with various complications such as venous rupture (38). Its use is limited only to cases in which CT does not offer adequate information. Computed tomography is the initial radiological method used to examine the possibility of malignancy. For a unilateral adenoma, the treatment consists of surgical removal. However, drugs can be used for patients who are either unwilling or not appropriate for surgery (36). These drugs include mineralocorticoid receptor antagonists such as eprenelone or spironolactone. In our case, there is a therapeutic dilemma since there is only one adrenal left. Thus, its resection will cause adrenal insufficiency. Furthermore, its imaging characteristics show no elements of malignancy. Since there are no precise guidelines about two concomitant secreting adrenal adenomas, it was decided to proceed to pharmaceutical treatment and not to surgery. We preferred a pharmaceutical approach with more than one antihypertensive drug, including spironolactone, in order to achieve a better pressure control and minimize adverse events. This will prevent adrenal insufficiency and lifetime oral administration of hydrocortisone to the patient. However, any change to the imaging characteristics compatible to malignancy or resistance to antihypertensive treatment will lead to adrenalectomy.

\section{CONCLUSION}

n conclusion, this report is noteworthy as the patient develops two different histological types of adrenal masses in a different location and time, a treated pheochromocytoma and a concomitant non-functioning cortical adrenal adenoma, which turned into aldosteronoma. The clinical elements of this case highlight the need of thorough preoperative search of hormone secretion and radiological findings of the adrenal mass, due to the possibility of malignancy and the side-effects of a possible hormone-secreting lesion. Adrenal insufficiency, with the need of continuous glucocorticoid replacement caused by adrenalectomy of both glands, is another issue that should be examined. Thus, we believe that all patients with adrenal mass should be evaluated according to the guidelines for the management of $\mathrm{Al}(2)$.

Conflicts of interest: none declared.

Financial support: none declared.

Patient's written informed consent for the publication of this case report and any

accompanying images was obtained. 


\section{R}

1. Young WF Jr. Clinical practice: The incidentally discovered adrenal mass. New Engl J Med 2007;356:601-610.

2. Zeiger MA, Thompson GB, Duh QY, et al. American Association of Clinical Endocrinologists; American Association of Endocrine Surgeons. The American Association of Clinical Endocrinologists and American Association of Endocrine Surgeons Medical Guidelines for the management of adrenal incidentalomas. Endocr Pract 2009;15 Suppl 1:1-20.

3. Jain MS. Adrenal incidentaloma: A puzzle for clinician. Indian J Endocrinol Metab 2013;17:59-63.

4. Santos P, Pimenta T, Taveira-Gomes A. Hereditary pheochromocytoma. Int J Surg Pathol 2014;22:393-400.

5. Hannemann A, Wallaschofski $H$. Prevalence of primary aldosteronism in patient's cohorts and in population-based studies--a review of the current literature. Horm Metab Res 2012;44:157-162.

6. Brunaud L, Duh QY. Aldosteronoma. Curr Treat Options Oncol 2002;3:327-333.

7. Douma S, Petidis K, Doumas M, et al. Prevalence of primary hyperaldosteronism in resistant hypertension: A retrospective observational study. Lancet 2008;371:1921-1926.

8. Calhoun DA, Jones D, Textor S, et al. American Heart Association Professional Education Committee. Resistant hypertension: Diagnosis, evaluation, and treatment: A scientific statement from the American Heart Association Professional Education Committee of the Council for High Blood Pressure Research. Circulation 2008;117:e510-e526.

9. Sato $\mathrm{H}$, Igarashi $\mathrm{H}$, Kishimoto $\mathrm{Y}$, et al. Combined tumor consisting of non-functioning adrenocortical adenoma and pheochromocytoma in the same gland. Int J Urol 2002;9:398-401.

10. Yoshida T, Setoguchi Y, Yamamoto K, et al. A case of a combined tumor consisting of adrenocortical adenoma and pheochromocytoma. Folia Endocrinol JPN 1997;73:463-469.

11. Cope O, Labbie JP, Raker JW, Bland EF. Pheochromocytoma and adrenal cortical adenoma. Report of a case with both tumors and discussion of their relation. J Clin Endocrinol Metab 1952;12:875-880.

12. Inoue J, Oishi S, Naomi S, et al. Pheochromocytoma associated with adrenocortical adenoma: Case report and literature review.

Endocrinol Jpn 1986;33:67-74.
13. Nagamura $Y$, Watanabe $K$, Nomoto $T$, et al. The coexistence of acromegaly, pituitary tumor, renal cell carcinoma, adrenal cortical adenoma and pheochromocytoma. Jpn J Cancer Clin 1978;24:370-372.

14. Tutsumi $Y$, Nakagami $Y$, Tsushima $T$, et al. A case of asymptomatic pheochromocytoma found by abdominal ultrasonography. Igakunoayumi 1987;140:816-820.

15. Huang WR, Ma WY, Tso AL, et al. Pheochromocytoma and adrenocortical adenoma in the same gland. J Clin Med Assoc 2007;70:289-293.

16. Zhang Y, Li H, Xiao J, et al. Bilateral adrenal tumors from different histology: Case report and literature review. Cell Biochem Biophys 2015;71:425-429.

17. Aso Y, Homma Y. A survey on incidental adrenal tumors in Japan. J Urol 1992;147:1478-1481.

18. Kojima M, Saitoh M, Itoh $H$, et al. Percutaneous biopsy for adrenal tumors using ultrasonically guided puncture. Tohoku J Exp Med 1994;172:333-343.

19. Kasprzak A, Nussdorfer GG, Malendowicz LK. Effects of unilateral adrenalectomy on the remaining adrenal cortex of adrenocorticotropic hormonetreated male and female hamsters. Acta Anat (Basel) 1989;136:291-295.

20. Fassnacht M, Arlt W, Bancos I, et al. Management of adrenal incidentalomas: European Society of Endocrinology Clinical Practice Guideline in collaboration with the European Network for the Study of Adrenal Tumors. Eur J Endocrinol 2016;175:G1-34.

21. Tsirlin A, Oo Y, Sharma R, et al. Pheochromocytoma: A review. Maturitas 2014;77:229-238.

22. Korobkin M, Brodeur FJ, Francis IR, et al. CT time-attenuation washout curves of adrenal adenomas and nonadenomas. AJR Am J Roentgenol 1998;170:747-752.

23. Szolar DH, Korobkin M, Reittner $P$, et al. Adrenocortical carcinomas and adrenal pheochromocytomas: Mass and enhancement loss evaluation at delayed contrast-enhanced CT. Radiology 2005;234:479-85.

24. Butt K, Ali S, Sattar Z, Ur Rahman A, Burt JR. Funny lumps, flaming pheo, and a broken heart: A rare case of pheochromocytoma. Cureus 2018;10:e3646.

25. Walther MM, Herring J, Enquist E, et al. von Recklinghausen's disease and pheochromocytomas.
J Urol 1999;162:1582-6.

26. Neumann HPH, Young WF Jr, Eng C. Pheochromocytoma and paraganglioma. N Engl J Med 2019;381:552.

27. Pawlu C, Bausch B, Reisch N, Neumann HP. Genetic testing for pheochromocytoma-associated syndromes. Ann Endocrinol 2005;66:178.

28. Ma C, Sun E, Lu B. Giant malignant pheochromocytoma in an elderly patient. Medicine (Baltimore) 2018;97:e0614.

29. Lenders JW, Duh QY, Eisenhofer G, et al. Pheochromocytoma and paraganglioma: An endocrine society clinical practice guideline. J Clin Endocrinol Metab 2014;99:1915-1942.

30. Young W Jr. Endocrine hypertention. In Williams textbook of endocrinology (eds Melmed S, Polonsky KS, Larsen PR, Kronenberg HM). Saunders/Elsevier, Philadelphia, $12^{\text {th }}$ edition, 2011, pp 545-580.

31. Jalil ND, Pattou FN, Combemale F, et al. Effectiveness and limits of preoperative imaging studies for the localisation of pheochromocytomas and paragangliomas: A review of 282 cases. French Association of Surgery (AFC), and The French Association of Endocrine Surgeons (AFCE). Eur J Surg 1998;164:23-28.

32. Brink I, Hoegerle S, Klisch J, Bley TA. Imaging of pheochromocytoma and paraganglioma. Fam Cancer 2005;4:61-68.

33. Sherlock M, Scarsbrook A, Abbas A, et al. Adrenal incidentaloma. Endocr Rev 2020;41:775-820.

34. Pantalone KM, Gopan T, Remer EM, et al. Change in adrenal mass size as a predictor of a malignant tumor. Endocr Pract 2010;16:577-587.

35. Unger N. Adrenal incidentaloma: Diagnostic and therapeutic concept from an endocrinological perspective. Chirurg 2019;90:3-8.

36. Funder JW, Carey RM, Mantero F, et al. The management of primary aldosteronism: Case detection, diagnosis, and treatment: An endocrine society clinical practice guideline. J Clin Endocrinol Metab 2016;101:1889-1916.

37. Ganguly A. Primary aldosteronism. N Engl J Med 1998;39:1828-1834.

38. Magill SB, Raff H, Shaker JL, et al. Comparison of adrenal vein sampling and computed tomography in the differentiation of primary aldosteronism. J Clin Endocrinol Metab 2001;86:1066-7101. 